\title{
Nitric oxide inhalation prompts weaning from the right ventricular assist device: Evaluation under continuous-flow biventricular assistance
}

\author{
George J. Endo, MD \\ Kazushi Kojima, MD \\ Kunihide Nakamura, MD, PhD \\ Yasunori Matsuzaki, MD, PhD \\ Toshio Onitsuka, MD, PhD
}

From Miyazaki Medical College, Miyazaki, Japan.

Received for publication Nov 27, 2001; revisions requested Jan 8, 2002; revisions received Jan 22, 2002; accepted for publication Feb 10, 2002.

Address for reprints: G. J. Endo, MD, the 2nd Department of Surgery, Miyazaki Medical College, 5200 Kihara, Kiyotake Miyazaki 889-1692 Japan (E-mail: sneakerg @ post1.miyazaki-med.ac.jp).

J Thorac Cardiovasc Surg 2002;124:739-49

Copyright (C) 2002 by The American Association for Thoracic Surgery

0022-5223/2002 \$35.00+0 $\quad \mathbf{1 2 / 1 / 1 2 4 6 6 9}$

doi:10.1067/mtc.2002.124669
Objective: The objective of this study was to investigate the effect of nitric oxide on the recovery of right heart function under global ischemia with a continuous-flow biventricular assist device support.

Methods: Fifteen piglets were divided into three groups: continuous-flow biventricular assist support only (control group), global ischemia with continuous-flow biventricular assist support (ischemia only group), and global ischemia with continuous-flow biventricular assist support plus nitric oxide inhalation (nitric oxide group). Two continuous-flow pumps were used as left and right ventricular assist devices. In the ischemic groups (ischemia only group and nitric oxide group), global ischemia was induced for 30 minutes and followed by a 6-hour reperfusion period; the nonischemic control group underwent a 6-hour perfusion period only. The left ventricular assist device was driven at a flow rate of more than 75 to $80 \mathrm{~mL} /(\mathrm{min}$. $\mathrm{kg}$ ). The right ventricular assist device was driven so as to sustain the left ventricular assist device flow, and the animal was weaned from it in accordance with the objective of cardiac recovery.

Results: Mean pulmonary arterial pressure remained low in the nitric oxide group (mean $23 \mathrm{~mm} \mathrm{Hg}$ ), whereas it rose from $19.9 \mathrm{~mm} \mathrm{Hg}$ to $39.3 \mathrm{~mm} \mathrm{Hg}$ in the ischemia group and to $26.2 \mathrm{~mm} \mathrm{Hg}$ in the control group. Mixed venous saturation was maintained at more than $60 \%$ in all cases. Although no piglets in the ischemia group were able to survive without continuous-flow biventricular assist support, the right ventricular assist device flow ratio (device flow/total systemic flow) in the nitric oxide group could be reduced in all cases, and it was possible to wean the piglets from right ventricular assist device support in 4 of 5 cases.

Conclusion: Inhalation of 40-ppm nitric oxide enabled smoother maintenance of the left ventricular assist device flow and prompted the weaning from right ventricular assist device support on continuous-flow biventricular assist.

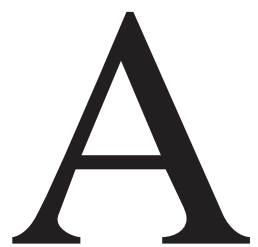

ventricular assist device (VAD) is often used to rescue patients with severe and uncontrollable heart failure. ${ }^{1}$ Typically, a left VAD (LVAD) is used for these patients, although biventricular mechanical assistance may be necessary during the early phase in some cases. Thus for many patients the need for right ventricular assistance is temporary. Therefore a management strategy for biventricular assistance that includes weaning from a right VAD (RVAD) is important. Few investigators have tried to determine management strategies for a continuous-flow biventricular assist device (CFBVAD), however, because the VADs commonly used clinically are based on pulsatile pumps.

The Journal of Thoracic and Cardiovascular Surgery • Volume 124, Number 4739 
Notably, the use of inhaled nitric oxide is attractive for its effect on right ventricular recovery through induction of pulmonary arterial dilatation,,$^{2-5}$ and it is used in combination with mechanical ventricular assistance in many medical facilities. ${ }^{6-9}$ The objective of this study was to investigate the effect of nitric oxide on the recovery of right heart function under global ischemia as a model of severe heart failure that needs LVAD support and temporal RVAD support, with CFBVAD support.

\section{Materials and Methods \\ Animals and Animal Care}

Fifteen male piglets $(32-43.5 \mathrm{~kg}$, mean $37.9 \mathrm{~kg})$ were used. All studies were conducted in the Experimental Animal Center of Miyazaki Medical College, and all experimental protocols were approved in advance by the animal care and use committee of this institution. The study complied with the "Principles of Laboratory Animal Care" developed by the National Society for Medical Research and the "Guide for the Care and Use of Laboratory Animals" prepared by the National Institutes of Animal Resources, National Research Council, and published by the National Academy Press, revised 1996.

\section{Preparation}

Piglets were treated in advance with an intramuscular injection of a cocktail of ketamine hydrochloride $(0.5 \mathrm{mg} / \mathrm{kg})$ and atropine sulfate $(0.5 \mathrm{mg})$. After induction of anesthesia by halothane, tracheotomy was performed. During surgery, anesthesia was maintained under mechanical ventilation with oxygen, nitrous oxide, and $0.5 \%$ to $2.0 \%$ halothane; the fraction of inspired oxygen was maintained at 0.5 to 0.8 . A venous line was established for continuous injection of Ringer's lactate solution $(10-15 \mathrm{~mL} /[\mathrm{kg}$. min]). Two catheters were placed for right ventricular pressure (RVP) and right atrial pressure (RAP) through the left internal thoracic vein, and an arterial catheter was placed in the internal thoracic artery for aortic pressure (AoP).

\section{Technique}

After systemic heparinization (100 U/kg bolus and 60-100 U/h continuous injection to maintain the activated coagulation time at 150-250 seconds), an LVAD and an RVAD were placed through a left thoracotomy. The inflow cannula of the LVAD was implanted into the left ventricle through the left atrium, and the outflow cannula was connected to a T-shaped glass tube implanted into the descending aorta. The inflow cannula of the RVAD was implanted into the right atrium, and the outflow cannula was connected to a conduit that was anastomosed to the pulmonary trunk. A catheter for left atrial pressure (LAP) and left ventricular pressure (LVP) was incorporated into the LVAD inflow conduit, and a catheter for pulmonary arterial pressure (PAP) was inserted into the pulmonary trunk through the RVAD outflow conduit. An ultrasonic flow probe (T201; Transonic Systems, Inc, Ithaca, NY) was placed around the base of the ascending aorta to monitor cardiac output (CO).

\section{Delivery of Nitric Oxide}

The stock gas (with a premixed concentration of 200-ppm nitric oxide in nitrogen) was supplied and delivered through one arm of a Y-connector that was connected directly to the endotracheal tube, where it was diluted with oxygen and anesthetic gas. The endotracheal nitric oxide concentration was maintained at $40 \mathrm{ppm}$ by flow control.

The exhaled gas was dehumidified using silica gel; the nitrogen oxide was absorbed onto soda lime and activated charcoal before exhaustion. At indeterminate time, nitric oxide concentration was confirmed in comparison with the determination curve, which was previously measured with various gas concentrations, by nitric oxide analyzer (ENO-20; EICOM Corp, Kyoto, Japan).

\section{Continuous-Flow Biventricular Assist Device Control Method}

Our control strategy was previously reported in detail. ${ }^{11-15}$ We set LVAD pump speed within the total assist, nonsucking range (safe range), with a target flow rate of 50 to $75 \mathrm{~mL} /(\mathrm{kg} \cdot \mathrm{min})(75$ $\mathrm{mL} /[\mathrm{kg} \cdot \mathrm{min}]$, ideally) to obtain the same flow in each group. Although this flow rate represents a slightly lower than usual level of support, it was determined on consideration of the mixed venous oxygen saturation $(>60 \%)$ in the ischemic group, which was barely able to maintain such a flow rate in this series. For consistency, the other groups were required to have no more than this flow. The RVAD speed was kept within the safe range, with RVAD flow controlled at the minimum level able to maintain the LVAD target flow.

\section{Protocol}

After the CFBVAD was in place, LVAD support was started, followed by induction of global ischemia by clamping of the base of the ascending aorta for 30 minutes. When cardiac dysfunction developed and the flow rate decreased, RVAD support was started. Nitrogen inhalation (control and ischemia groups) or 40-ppm nitric oxide inhalation (nitric oxide group) was performed, at the same flow rate, on commencement of RVAD support (Figure 1). If no cardiac recovery had appeared by 2 hours after reperfusion, electric defibrillation was performed (10-60 J). In addition, if no response was achieved with $60-\mathrm{J}$ stimulation, a retry was performed after 15 minutes.

Hemodynamic data were collected every 5 minutes after the start of LVAD support. To detect native cardiac function, data were sampled with the pumps transiently stopped for 30 seconds, at 1-hour intervals after unclamping. All animals were killed under deep anesthesia and potassium chloride injection after more than 6 hours of observation. To maintain the preload of RVAD, Ringer's lactate solution was injected at the rate of $120 \mathrm{~mL} / \mathrm{h}$ in all groups, to make the conditions of preload the same with each animal.

\section{Sampling and Recording of Data}

AoP, PAP, LVP, LAP, RVP, RAP, pump speeds (LVAD and RVAD), CO, and pump flow rates were measured. Left and right ventricular instantaneous pressures ( $\mathrm{LV} \mathrm{dp/dt}$ and $\mathrm{RV} \mathrm{dp} / \mathrm{dt}$, respectively) and left and right ventricular end-diastolic pressures (LVEDP and RVEDP, respectively) without biventricular VAD (BVAD) support were sampled during transient CFBVAD inactivation by clamping the outflow cannulas, with derived quantities calculated from the waveforms. Blood gas values (arterial and venous) were measured every 30 minutes. 




Figure 1. Protocol of this study. After CFBVAD was in place, global ischemia was induced by clamping base of ascending aorta for $\mathbf{3 0}$ minutes in ischemia groups (group I) and nitric oxide group (group N). Nitrogen (control group [group C] and ischemia group) or $\mathbf{4 0}-\mathrm{ppm}$ nitric oxide (NO, nitric oxide group) inhalation was administered, at same flow rate, when RVAD support was initiated. Hemodynamic data were collected every 5 minutes after start of LVAD support. In addition, blood was sampled every hour for blood chemistry analysis.

All values were digitized by a personal computer (EN6450; COMPAQ Japan, Tokyo, Japan) running a data collection program (Labview 5.0; National Instruments, Austin, Tex). Pressures were recorded through a polygraph (EP1102; NEC, Tokyo, Japan), whereas motor current and pump speed were entered directly.

The circulating blood was sampled every hour to determine the cell count and the blood chemistry profile as follows: blood urea nitrogen and creatinine were used as parameters to assess renal function; alanine aminotransferase and total bilirubin values were used to assess liver injury; creatine phosphokinase MB isoenzyme and troponin $\mathrm{T}$ were used to assess myocardial injury; free hemoglobin was used to assess hemolysis; aspartate aminotransferase and lactate dehydrogenase were used to assess non-specific cell injury; and methemoglobin was used to assess red blood cell injury by nitric oxide.

Lung tissue was collected to assess lung edema. Tissue resected from the apex was fixed by formalin and microscopic assessment was performed after hematoxylin and eosin staining. For another tissue sample from the lower lobe, the wet/dry ratio was determined from the masses before and after drying under the condition of 100 for 72 hours. ${ }^{16}$

\section{Statistical Analysis}

All results were expressed as means and standard deviations and analyses of data were performed with commercial statistical software (STATVIEW 5.0; SAS Institute, Inc, Cary, NC). Analysis of variance was used to compare measurements at baseline with those taken during the postreperfusion phase. The Fisher protected least significant difference was used to test the significance of differences.

\section{Results}

Four phases determined by pump flow and cardiac condition were observed, especially in the nitric oxide group (Figure 2, C). The first observation phase was during ventricular fibrillation (Vf phase); during this phase no native $\mathrm{CO}$ was seen. The second observation phase followed electric defibrillation, in which low native output was seen (weak contraction phase). The third phase was characterized by an excess of left pump flow (LPF) over right pump flow (RPF; RVAD weaning phase). The last observation phase took place after RVAD support was stopped (no-RVAD phase). During global ischemia and early after reperfusion (effectively the Vf phase), native beating disappeared and $\mathrm{CO}$ was almost zero, so that the animal could not live without CFBVAD support. In this phase the ischemia group required that LVAD speed be increased to the maximum possible before sucking occurred, and to secure sufficient LPF it needed higher RPF to provide sufficient preload to the LVAD because, on measurement, RPF appeared to exceed LPF.

Electric defibrillation was performed 2 hours after reperfusion in 9 of 10 cases in the ischemic groups ( 5 in ischemia group, 4 in nitric oxide group). As the cardiac function 


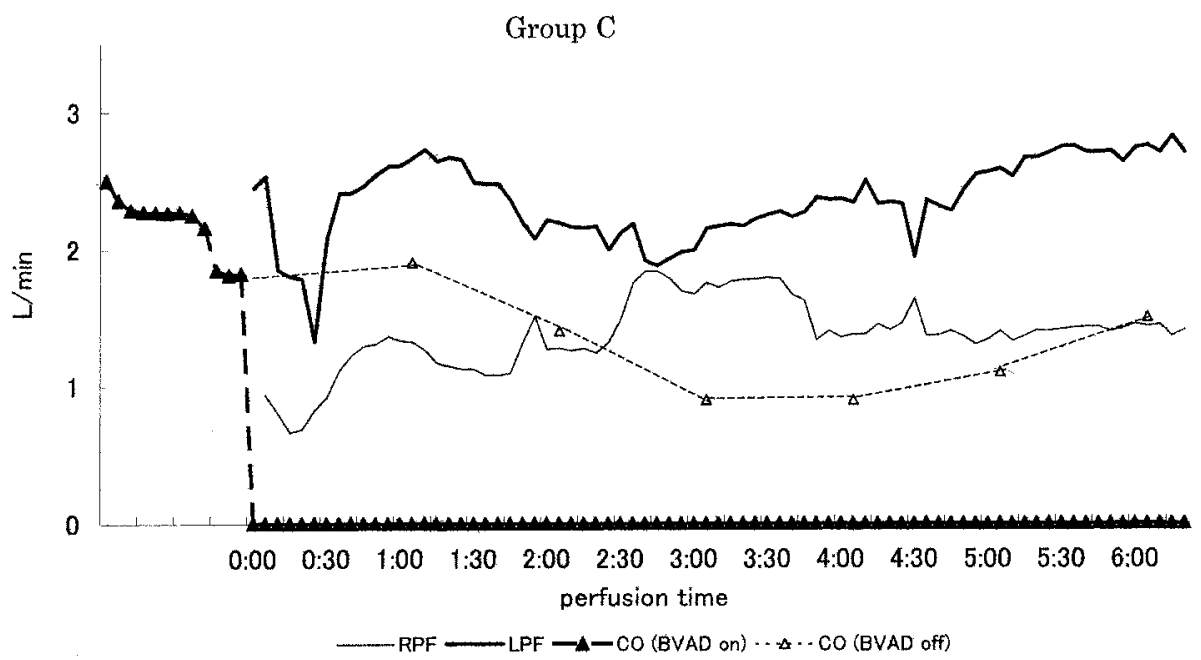

Group I
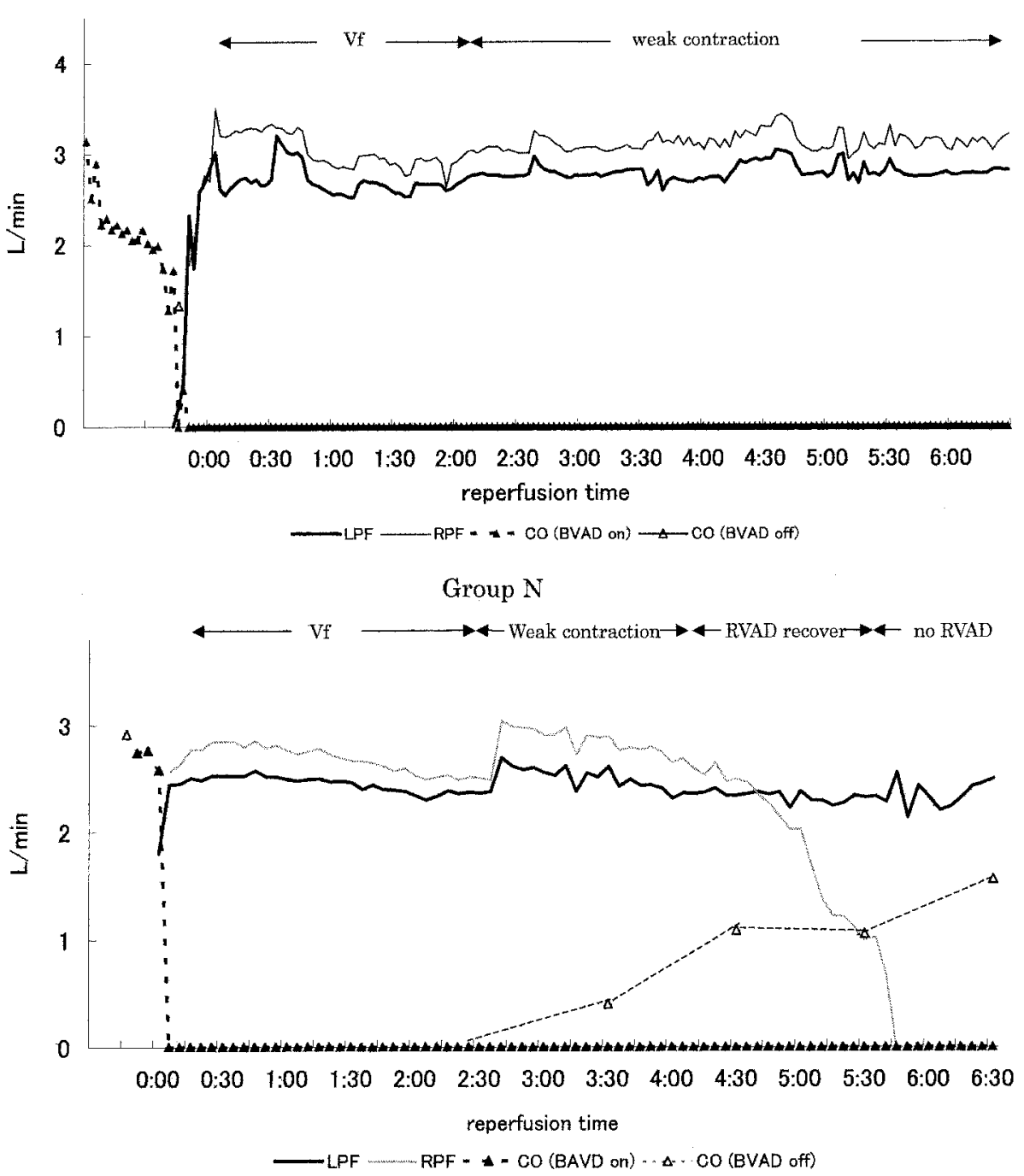

Figure 2. Time course of pump flow in each group. A, Control group (group C). B, Ischemia group (group I). C, Nitric oxide group (group $\mathbf{N}$ ). 
TABLE 1. Hemodynamic canges during BVAD support

\begin{tabular}{|c|c|c|c|c|c|}
\hline Group & BVAD on & $1 \mathrm{~h}$ & $2 \mathrm{~h}$ & $3 \mathrm{~h}$ & $6 \mathrm{~h}$ \\
\hline \multicolumn{6}{|c|}{ On-AoP (mm Hg) } \\
\hline Control & $51.2 \pm 12.5$ & $56.0 \pm 5.4$ & $54.0 \pm 6.5$ & $51.9 \pm 6.6$ & $64.2 \pm 14.5$ \\
\hline Ischemia & $50.2 \pm 9.4$ & $56.0 \pm 10.7$ & $55.3 \pm 9.4$ & $51.3 \pm 7.5$ & $51.2 \pm 11.3$ \\
\hline Nitric oxide & $57.5 \pm 10.9$ & $57.2 \pm 8.9$ & $60.0 \pm 9.4$ & $59.5 \pm 7.5$ & $60.5 \pm 15.7$ \\
\hline \multicolumn{6}{|c|}{ On-LVP (mm Hg) } \\
\hline Control & $26.4 \pm 15.2$ & $19.9 \pm 5.9$ & $16.5 \pm 5.0$ & $11.0 \pm 3.2$ & $26.1 \pm 8.4$ \\
\hline Ischemia & $19.4 \pm 8.4$ & $12.6 \pm 14.5$ & $14.5 \pm 10.6$ & $14.7 \pm 11.6$ & $13.5 \pm 10.2^{*}$ \\
\hline Nitric oxide & $13.4 \pm 6.5$ & $15.6 \pm 7.9$ & $16.0 \pm 4.0$ & $15.3 \pm 4.0$ & $23.4 \pm 3.9$ \\
\hline \multicolumn{6}{|c|}{ On-LAP (mm Hg) } \\
\hline Control & $9.0 \pm 2.7$ & $7.6 \pm 3.5$ & $6.4 \pm 2.2$ & $6.4 \pm 2.2$ & $8.7 \pm 1.8$ \\
\hline Ischemia & $4.3 \pm 3.9$ & $14.1 \pm 10.8$ & $12.4 \pm 11.0$ & $10.0 \pm 9.7$ & $11.4 \pm 8.6$ \\
\hline Nitric oxide & $8.2 \pm 2.1$ & $8.8 \pm 3.9$ & $10.2 \pm 2.9$ & $9.8 \pm 6.5$ & $10.7 \pm 3.3$ \\
\hline \multicolumn{6}{|c|}{ On-PAP (mm Hg) } \\
\hline Control & $25.8 \pm 8.2$ & $26.2 \pm 6.1$ & $28.4 \pm 9.2$ & $27.8 \pm 9.7$ & $27.9 \pm 3.1$ \\
\hline Ischemia & $25.5 \pm 10.7$ & $38.8 \pm 6.2 \dagger$ & $7.8 \pm 3.2 \dagger$ & $38.4 \pm 5.5 \dagger$ & $38.6 \pm 8.4 \dagger$ \\
\hline Nitric oxide & $25.3 \pm 6.2$ & $23.3 \pm 2.9^{*}$ & $5.1 \pm 2.3^{*}$ & $23.7 \pm 6.0^{*}$ & $24.9 \pm 6.4^{*}$ \\
\hline \multicolumn{6}{|c|}{ On-RVP $(\mathrm{mm} \mathrm{Hg})$} \\
\hline Control & $14.5 \pm 2.5$ & $15.7 \pm 3.3$ & $15.4 \pm 2.7$ & $16.0 \pm 5.3$ & $13.8 \pm 6.5$ \\
\hline Ischemia & $13.6 \pm 2.0$ & $7.5 \pm 2.7 \dagger$ & $7.4 \pm 2.8 \dagger$ & $9.2 \pm 2.6 \dagger$ & $7.8 \pm 2.7 \dagger$ \\
\hline Nitric oxide & $13.5 \pm 2.1$ & $7.5 \pm 6.6 \dagger$ & $7.1 \pm 6.4 \dagger$ & $11.1 \pm 4.7$ & $19.0 \pm 3.6^{*}$ \\
\hline \multicolumn{6}{|c|}{ On-RAP (mm Hg) } \\
\hline Control & $3.3 \pm 2.6$ & $3.1 \pm 2.2$ & $3.5 \pm 1.6$ & $4.1 \pm 2.1$ & $7.9 \pm 2.7$ \\
\hline Ischemia & $4.4 \pm 1.8$ & $2.0 \pm 1.5$ & $3.7 \pm 1.3$ & $4.5 \pm 2.5$ & $5.2 \pm 6.0$ \\
\hline Nitric oxide & $3.4 \pm 1.6$ & $3.2 \pm 1.5$ & $2.8 \pm 1.9$ & $2.6 \pm 1.9$ & $7.1 \pm 1.9$ \\
\hline \multicolumn{6}{|l|}{ LPF (L/min) } \\
\hline Control & $2.2 \pm 0.6$ & $2.5 \pm 0.5$ & $2.1 \pm 0.5$ & $2.0 \pm 0.4$ & $2.4 \pm 0.3$ \\
\hline Ischemia & $2.1 \pm 0.5$ & $2.3 \pm 0.6$ & $2.6 \pm 1.1$ & $2.4 \pm 0.9$ & $2.3 \pm 0.9$ \\
\hline Nitric oxide & $2.5 \pm 0.3$ & $2.4 \pm 0.3$ & $2.3 \pm 0.4$ & $2.3 \pm 0.4$ & $2.4 \pm 0.3$ \\
\hline \multicolumn{6}{|c|}{ Systemic vascular resistance $\left(\mathrm{dyne} \cdot \mathrm{s} / \mathrm{cm}^{5}\right)$} \\
\hline Control & $1937.7 \pm 216.6$ & $1693.4 \pm 298.3$ & $1858.5 \pm 318.0$ & $1569.5 \pm 430.9$ & $1789.4 \pm 242.1$ \\
\hline Ischemia & $1738.2 \pm 390.9$ & $1881.0 \pm 190.6$ & $1901.6 \pm 619.0$ & $1696.5 \pm 457.2$ & $1905.7 \pm 698.9$ \\
\hline Nitric oxide & $1932.7 \pm 535.7$ & $1743.7 \pm 139.0$ & $1970.1 \pm 147.4$ & $1957.6 \pm 94.6$ & $1681.2 \pm 615.2$ \\
\hline \multicolumn{6}{|c|}{ PVR (dyne $\cdot \mathrm{s} / \mathrm{cm}^{5}$ ) } \\
\hline Control & $679.4 \pm 335.9$ & $612.8 \pm 270.7$ & $863.7 \pm 443.2$ & $941.9 \pm 400.1$ & $646.8 \pm 201.6$ \\
\hline Ischemia & $702.8 \pm 463.9$ & $867.0 \pm 325.8$ & $948.9 \pm 361.6$ & $1022.7 \pm 282.1$ & $1097.9 \pm 445.2 \dagger$ \\
\hline Nitric oxide & $539.7 \pm 213.3$ & $519.3 \pm 164.5$ & $535.7 \pm 174.3$ & $560.8 \pm 274.1^{*}$ & $453.5 \pm 211.8^{*}$ \\
\hline
\end{tabular}

${ }^{*} P<.05$ versus ischemia alone group.

$\dagger P<.05$ versus control group.

gradually recovered during this procedure, the relative magnitude of pump flow rates was able to reverse (LPF greater than RPF). By controlling a CFBVAD as described previously, RPF was decreased gradually without loss of LPF, and the piglets were weaned from the RVAD support almost automatically in all cases in the nitric oxide group.

In the ischemia group, the Vf phase and the weak contraction phase were observed but the other phases were not because of poor recovery of cardiac function. In the control group, in which ischemia was not induced, only the RVAD weaning phase and the no-RVAD phase were observed (Figure $2 A$ and $B$ ).

Because significant reddening of the lung was observed just after global ischemia and RVAD support in the ischemia group, the existence of lung congestion was suspected. This color change improved slightly during the 6 hours of observation but did not fade completely. Such color change was rarely observed in the control and nitric oxide groups.

\section{Hemodynamic Parameters}

The time courses of the hemodynamic parameters are shown in Table 1 and Figure 3. AoP with CFBVAD support was maintained at $56.0 \pm 11.1 \mathrm{~mm} \mathrm{Hg}$ in the control group, $54.5 \pm 11.4 \mathrm{~mm} \mathrm{Hg}$ in the ischemia group, and $60.5 \pm 7.9$ $\mathrm{mm} \mathrm{Hg}$ in the nitric oxide group, all of which values were similar to the values before support $(56.0 \pm 11.1 \mathrm{~mm} \mathrm{Hg}$, $55.7 \pm 10.6 \mathrm{~mm} \mathrm{Hg}$, and $59.4 \pm 7.1 \mathrm{~mm} \mathrm{Hg}$, respectively). There was no significant difference between the groups at any time.

Although its initial value was the same, PAP rose during the process of equipping $\mathrm{BVAD}$, in each group (control 


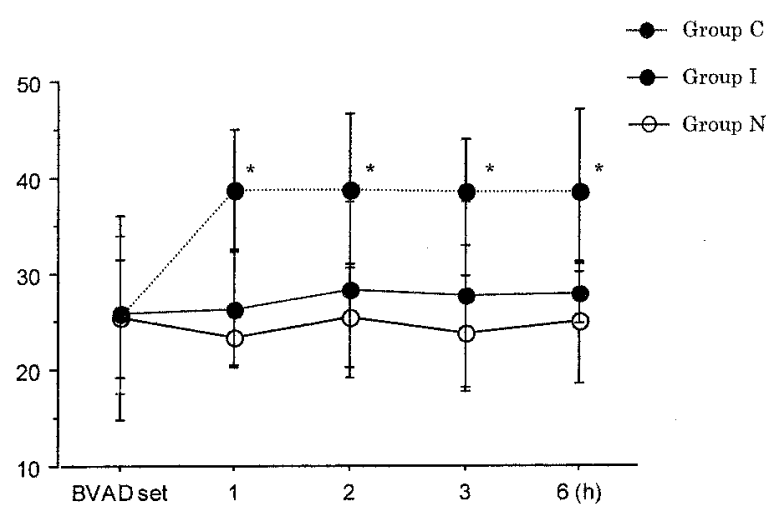

A

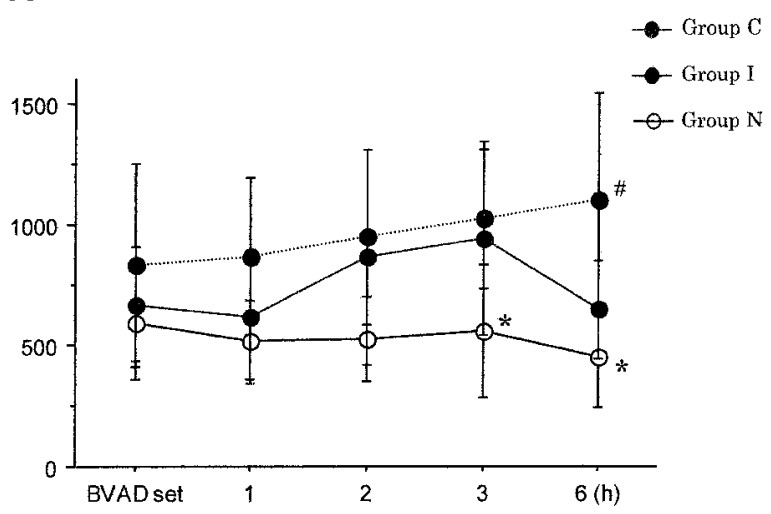

B

Figure 3. Time courses of on-PAP (A) and PVR (B). Inhalation of 40-ppm nitric oxide during CFBVAD support reduced PVR and PAP immediately in nitric oxide group (group $N$ ), whereas it remained higher level in ischemia group (group I). Group C, Control group.

$25.8 \pm 8.2 \mathrm{~mm} \mathrm{Hg}$, ischemia $25.5 \pm 10.7 \mathrm{~mm} \mathrm{Hg}$, and nitric oxide $25.3 \pm 6.2 \mathrm{~mm} \mathrm{Hg}$ ). In the control group, PAP during reperfusion was maintained at an average of $26.2 \pm 6.1 \mathrm{~mm}$ $\mathrm{Hg}$. In the ischemia group, it rose to $38.8 \pm 6.2 \mathrm{~mm} \mathrm{Hg}$ after 1 hour of reperfusion and was maintained at an average of $39.2 \pm 6.4 \mathrm{~mm} \mathrm{Hg}$ during 6 hours of reperfusion. In the nitric oxide group, PAP rose to $23.2 \pm 6.1 \mathrm{~mm} \mathrm{Hg}$ in the first hour of reperfusion and was maintained at an average of $24.2 \pm 4.9 \mathrm{~mm} \mathrm{Hg}$ during the 6-hour reperfusion. After 6 hours of reperfusion, a significant difference in PAP was detected between the groups (control vs ischemia $P<$ .0001 , ischemia vs nitric oxide $P<.0001$, and control vs nitric oxide $P<.005$; Figure 3, A).

Pulmonary vascular resistance (PVR) gradually increased after global ischemia and 6-hour reperfusion, from $702.8 \pm 463.9$ dyne $\cdot \mathrm{s} \cdot \mathrm{cm}^{-5}$ at BVAD equipping period to $994.9 \pm 410.2$ dyne $\cdot \mathrm{s} \cdot \mathrm{cm}^{-5}$ in the ischemia group. That of the nitric oxide group decreased, from $539.7 \pm 213.3$ dyne $\cdot \mathrm{s} \cdot \mathrm{cm}^{-5}$ to $366.1 \pm 94.5$ dyne $\cdot \mathrm{s} \cdot \mathrm{cm}^{-5}$ after 6 hours of nitric oxide inhalation (Figure 3, $B$ ).

Pump flow $(2.2 \pm 0.4 \mathrm{~L} / \mathrm{min}$ in the control group, $2.4 \pm$
$0.8 \mathrm{~L} / \mathrm{min}$ in the ischemia group, and $2.4 \pm 0.3 \mathrm{~L} / \mathrm{min}$ in nitric oxide group) remained at $80 \%$ to $85 \%$ of basal $\mathrm{CO}$ $(2.7 \pm 0.7 \mathrm{~L} / \mathrm{min}, 2.7 \pm 0.5 \mathrm{~L} / \mathrm{min}$, and $2.9 \pm 0.5 \mathrm{~L} / \mathrm{min}$, respectively). To maintain the target flow in the ischemia group, however, it was necessary to increase the pump speed to the vicinity of the point at which sucking occurred, to maximize LVAD and RVAD flow rate.

The time courses of native cardiac function estimated during transient CFBVAD inactivation and the support rate of CFBVAD are shown in Table 2 and Figure 4. CO rarely recovered after global ischemia in the ischemia group. In contrast, whereas $\mathrm{CO}$ in the nitric oxide group showed a decline similar to that in the ischemia group at the global ischemia phase, this was only temporary, and it then gradually increased with reperfusion until recovery was almost that of the control group at 5.5 hours after reperfusion (Figure 4, A).

Reduced LV dp/dt, caused by global ischemia, showed a step increase after defibrillation in the ischemic groups. Although in the ischemia group it gradually decreased through reperfusion, in the nitric oxide group it increased to about $80 \%$ of baseline or that of the control group $(P<.05$; Figure 4, $B$ ). LVEDP in both ischemic groups declined from global ischemia to defibrillation and increased after defibrillation. The rate of decline was smaller in the nitric oxide group, but no significant difference was observed when compared with the ischemia group (Figure $4, C$ ). RV dp/dt showed an increase-and-decrease pattern that was similar to that of LV dp/dt $(P<.05$; Figure $4, D)$. RVEDP in both ischemic groups declined from global ischemia to defibrillation and increased after the defibrillation. The rate of decline was smaller in the nitric oxide group, but no significant difference was observed when compared with the ischemia group (Figure 4, E).

The consumption and supply of oxygen are shown in Table 3. In all groups, mixed venous oxygen saturation $\left(\mathrm{SvO}_{2}\right)$, which was relied on as a parameter for systemic oxygen supply, was maintained at greater than $60 \%$, this being considered as indicative that pump flow was sufficient for systemic oxygen demand, in all phases. However, to keep the $\mathrm{SvO}_{2}$ above $60 \%$, the fraction of inspired oxygen had to be raised to $0.6 \pm 1.0$ in the ischemia group, despite the requirement of the protocol for the fraction of inspired oxygen of the nitric oxide group to be 0.5 .

In addition, although spontaneous micturition was not observed in the ischemia group, it was observed in all cases in the nitric oxide group at 4.5 to 5.5 hours after reperfusion.

\section{Blood Chemistry Data}

Blood urea nitrogen, creatinine, C3a, aspartate aminotransferase, and lactate dehydrogenase increased gradually in all groups, but no significant differences were observed between groups. Alanine aminotransferase and total bilirubin 
TABLE 2. Time courses of native cardiac function

\begin{tabular}{|c|c|c|c|c|c|c|}
\hline Group & Baseline & BVAD on & $1 \mathrm{~h}$ & $2 \mathrm{~h}$ & $3 \mathrm{~h}$ & $6 \mathrm{~h}$ \\
\hline \multicolumn{7}{|l|}{ CO (L/min) } \\
\hline Control & $2.7 \pm 0.7$ & $2.4 \pm 0.4$ & $1.9 \pm 0.4$ & $1.4 \pm 0.2$ & $0.9 \pm 0.5$ & $1.5 \pm 0.8$ \\
\hline Ischemia & $2.7 \pm 0.6$ & $2.1 \pm 0.5$ & - & - & $0.1 \pm 0.1^{*}$ & $0.3 \pm 0.5^{*}$ \\
\hline Nitric oxide & $3.0 \pm 0.8$ & $2.4 \pm 0.3$ & $0.5 \pm 0.9$ & $0.2 \pm 0.4$ & $0.7 \pm 0.3 \dagger$ & $1.3 \pm 0.2 \dagger$ \\
\hline \multicolumn{7}{|l|}{ LVEDP (mm Hg) } \\
\hline Control & $10.3 \pm 4.1$ & $10.4 \pm 2.4$ & $10.6 \pm 0.0$ & $12.1 \pm 0.7$ & $12.0 \pm 1.4$ & $14.2 \pm 2.3$ \\
\hline Ischemia & $13.1 \pm 2.8$ & $1.5 \pm 7.7$ & - & - & $11.1 \pm 10.5$ & $12.6 \pm 11.7$ \\
\hline Nitric oxide & $11.7 \pm 4.2$ & $2.8 \pm 8.6$ & $6.2 \pm 10.7$ & $8.5 \pm 11.2$ & $16.1 \pm 1.9$ & $20.0 \pm 2.6$ \\
\hline \multicolumn{7}{|l|}{ RVEDP (mm Hg) } \\
\hline Control & $6.8 \pm 0.1$ & $6.5 \pm 0.2$ & $6.3 \pm 0.4$ & $8.2 \pm 0.5$ & $8.1 \pm 4.2$ & $7.0 \pm 1.8$ \\
\hline Ischemia & $7.0 \pm 2.8$ & $3.0 \pm 6.8$ & - & - & $11.6 \pm 4.2$ & $8.2 \pm 7.5$ \\
\hline Nitric oxide & $4.0 \pm 4.3$ & $0.2 \pm 7.8$ & $3.3 \pm 5.7$ & $3.6 \pm 6.2$ & $6.8 \pm 1.1$ & $7.1 \pm 4.4$ \\
\hline \multicolumn{7}{|c|}{ LV dp/dt (mm Hg/s) } \\
\hline Control & - & $495.5 \pm 342.4$ & - & $427.5 \pm 128.8$ & $356.3 \pm 145.9$ & $588.0 \pm 211.5$ \\
\hline Ischemia & - & $483.5 \pm 185.1$ & - & - & $149.7 \pm 95.9$ & $82.5 \pm 100.6^{*}$ \\
\hline Nitric oxide & - & $709.3 \pm 80.8$ & - & $92.1 \pm 159.6$ & $215.6 \pm 131.8$ & $461.6 \pm 58.3$ \\
\hline \multicolumn{7}{|c|}{$\mathrm{RV} \mathrm{dp} / \mathrm{dt}(\mathrm{mm} \mathrm{Hg} / \mathrm{s})$} \\
\hline Control & - & $256.5 \pm 91.0$ & - & $274.5 \pm 17.9$ & $257.2 \pm 27.0$ & $260.5 \pm 72.3$ \\
\hline Ischemia & - & $257.5 \pm 133.6$ & - & - & $55.8 \pm 12.1$ & $48.0 \pm 19.0^{*}$ \\
\hline Nitric oxide & - & $298.3 \pm 2.9$ & - & $77.0 \pm 133.4$ & $187.4 \pm 1.6$ & $250.4 \pm 25.4 \dagger$ \\
\hline \multicolumn{7}{|c|}{ Off-LAP $(\mathrm{mm} \mathrm{Hg})$} \\
\hline Control & $9.4 \pm 4.1$ & $12.0 \pm 4.3$ & $10.0 \pm 1.1$ & $10.9 \pm 1.7$ & $10.9 \pm 3.8$ & $15.5 \pm 2.5$ \\
\hline Ischemia & $7.9 \pm 3.7$ & $11.5 \pm 3.2$ & $7.2 \pm 9.8$ & $7.9 \pm 6.3$ & $10.3 \pm 8.2$ & $11.6 \pm 4.3$ \\
\hline Nitric oxide & $7.7 \pm 2.6$ & $9.6 \pm 1.2$ & $10.3 \pm 3.1$ & $8.5 \pm 2.8$ & $8.1 \pm 5.2$ & $16.8 \pm 4.5$ \\
\hline \multicolumn{7}{|c|}{ Off-LVP (mm Hg) } \\
\hline Control & $40.1 \pm 10.4$ & $46.8 \pm 5.9$ & $40.4 \pm 7.8$ & $36.8 \pm 9.2$ & $33.2 \pm 13.3$ & $42.0 \pm 12.4$ \\
\hline Ischemia & $36.7 \pm 3.7$ & $33.5 \pm 12.7$ & $18.8 \pm 9.9^{*}$ & $12.9 \pm 8.5^{*}$ & $20.4 \pm 3.5$ & $17.2 \pm 3.6^{*}$ \\
\hline Nitric oxide & $38.2 \pm 5.2$ & $49.8 \pm 5.4$ & $25.8 \pm 10.8$ & $20.2 \pm 6.8^{*}$ & $24.1 \pm 8.6$ & $33.6 \pm 12.3$ \\
\hline \multicolumn{7}{|c|}{ Off-RAP (mm Hg) } \\
\hline Control & $2.0 \pm 1.3$ & $2.0 \pm 0.8$ & $3.8 \pm 2.2$ & $3.7 \pm 2.2$ & $5.1 \pm 1.5$ & $9.0 \pm 5.6$ \\
\hline Ischemia & $2.2 \pm 1.2$ & $2.4 \pm 0.6$ & $8.3 \pm 7.9$ & $8.3 \pm 4.8$ & $9.6 \pm 4.8$ & $7.8 \pm 6.9$ \\
\hline Nitric oxide & $1.8 \pm 1.8$ & $2.2 \pm 0.6$ & $6.4 \pm 5.1$ & $6.1 \pm 4.8$ & $3.6 \pm 2.0 \dagger$ & $7.0 \pm 2.2$ \\
\hline \multicolumn{7}{|c|}{ Off-RVP (mm Hg) } \\
\hline Control & - & $16.7 \pm 2.8$ & $18.4 \pm 2.7$ & $17.8 \pm 2.6$ & $14.8 \pm 1.8$ & $17.5 \pm 2.4$ \\
\hline Ischemia & - & $13.9 \pm 5.9$ & $12.3 \pm 5.6$ & $12.5 \pm 3.3$ & $14.4 \pm 3.4$ & $13.6 \pm 3.6$ \\
\hline Nitric oxide & - & $20.8 \pm 2.1$ & $13.2 \pm 4.9$ & $11.3 \pm 3.7$ & $10.9 \pm 1.8$ & $18.2 \pm 4.1$ \\
\hline
\end{tabular}

${ }^{*} P<.05$ versus control group.

$\dagger P<.05$ versus ischemia only group.

values remained within the normal range in all groups, suggesting that no liver dysfunction existed.

Creatine phosphokinase MB isoenzyme and troponin $\mathrm{T}$ increased in each group just after the BVAD was implanted. Although these remained almost constant throughout the reperfusion time in the control group, they gradually increased in the ischemic groups (ischemia and nitric oxide groups). Thus the degree of myocardial damage induced by myocardial ischemia was similar in both ischemic groups.

Free hemoglobin, which was used as a parameter for hemolysis, increased in each group, the value for the nitric oxide group lying between those of the control and ischemia groups. However, although there was a significant difference between the ischemia group and the control group, differences between the nitric oxide group and the others were not significant.
Methemoglobin remained less than $1.2 \mathrm{mg} / \mathrm{dL}(10 \%-$ $15 \%$ of hemoglobin value) in all cases in the nitric oxide group.

\section{Pathologic Findings in Lung}

As a matter of convenience, pathologic findings in resected lung tissue were classified into three degrees: mild, no edema or only intravascular congestion in the section; moderate, interstitial edema in the section; and severe, interstitial edema and intra-alveolar effusion or hemorrhage the section. Whereas almost normal findings were made in the control group ( 3 mild and 2 moderate), frank interstitial edema was observed in the ischemia group (1 mild, 2 moderate, and 2 severe). On the other hand, despite ischemic conditions, no interstitial edema was found in the nitric oxide group ( 2 mild and 3 moderate). 

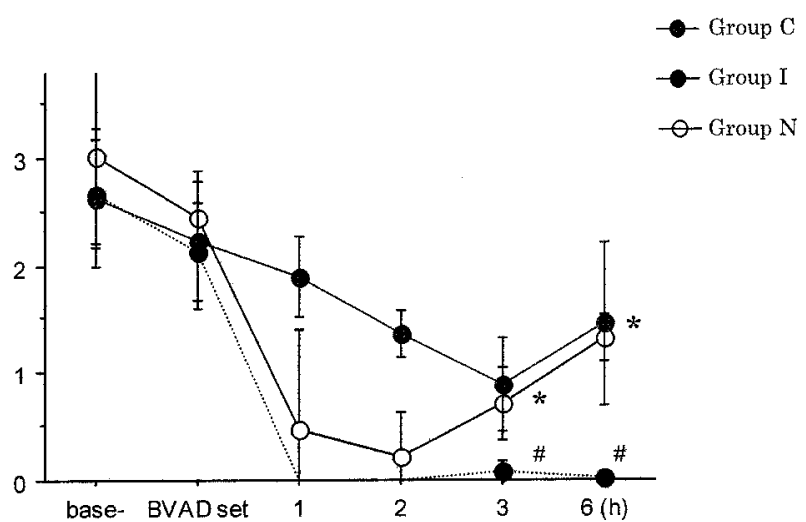

A
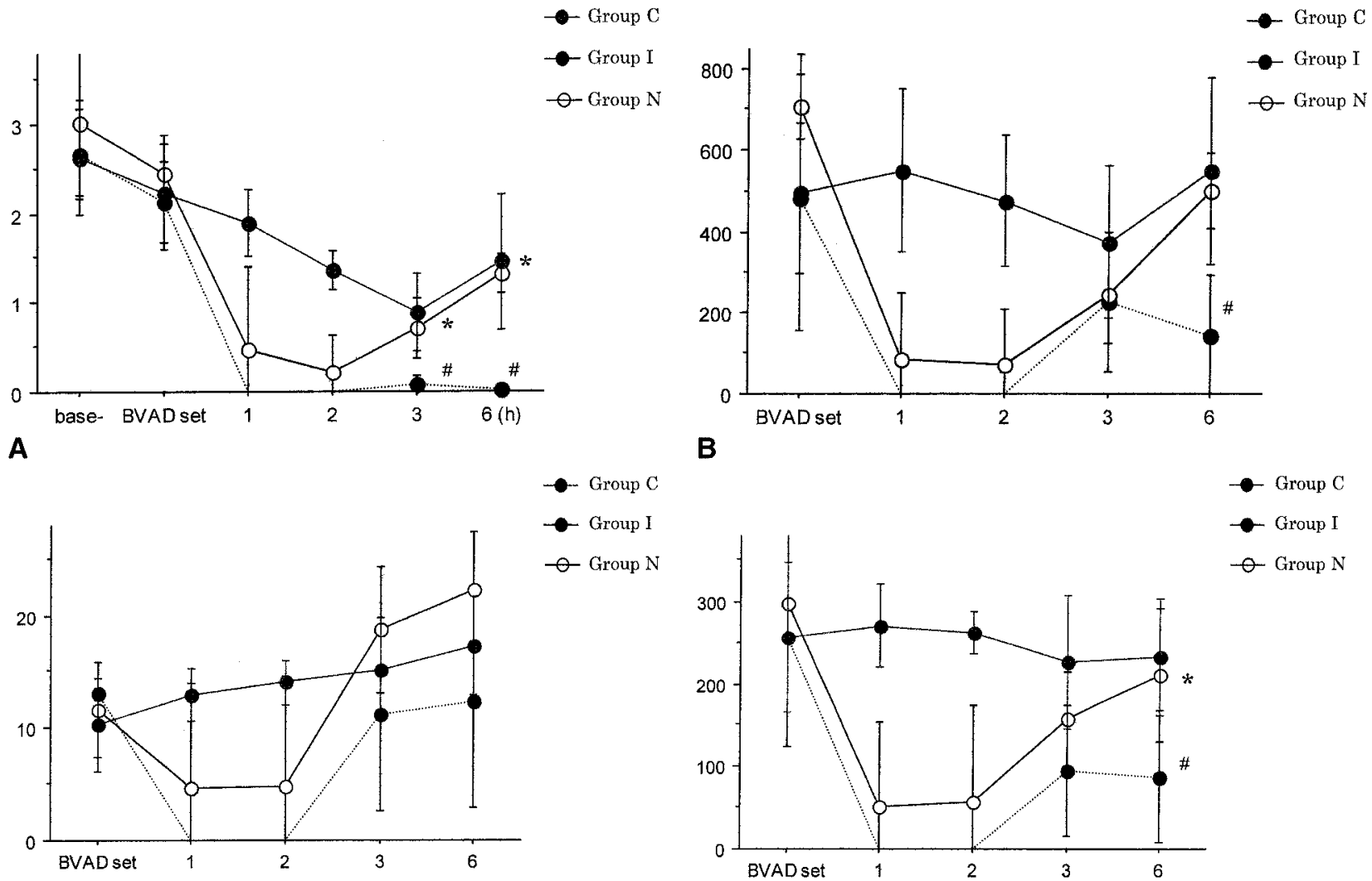

- Group C

B

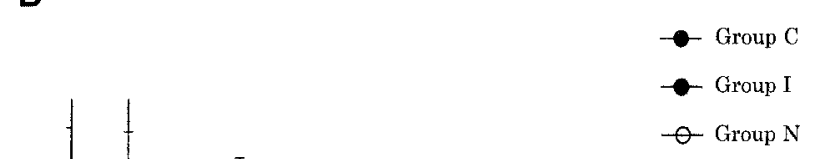

C

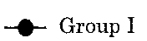

$\odot \operatorname{Group} N$

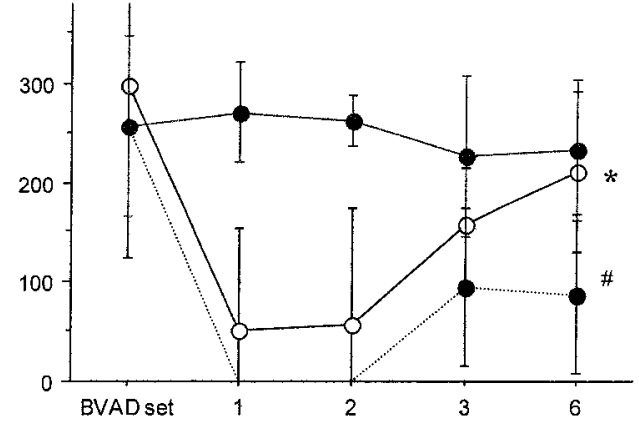

D

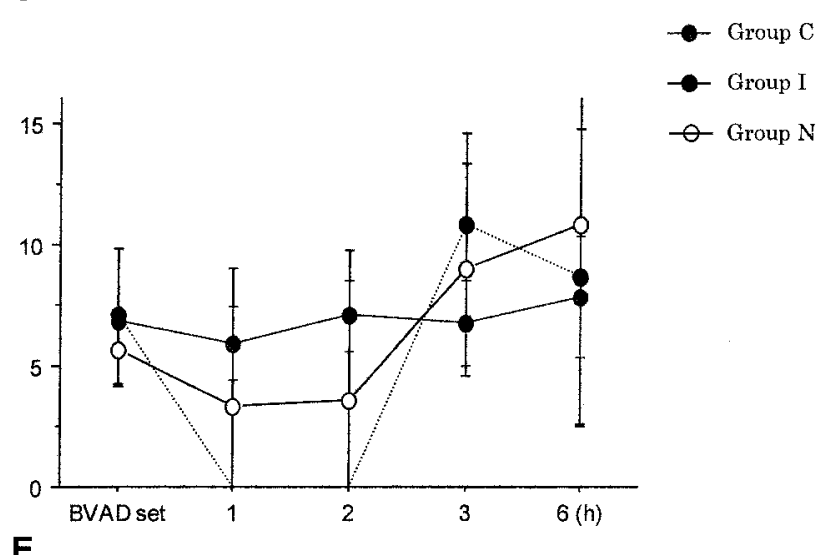

E

Figure 4. Time courses of hemodynamic changes based on native circulation. A, CO. B, Maximum LV dp/dt (LV max $d p / d t)$. C, LVEDP. D, Maximum RV dp/dt (RV max dp/dt). E, RVEDP. Within observation time (6 hours), recovery of CO was not observed in ischemia group but was observed in nitric oxide group, in which $\mathrm{CO}$ recovered to almost same grade as control group. Moreover, although LVEDP and RVEDP showed no significant differences in each group, recoveries of $\mathrm{LV} \mathrm{dp} / \mathrm{dt}$ and $\mathrm{RV} \mathrm{dp} / \mathrm{dt}$ at same grade were observed, without time lag.

\section{Wet/Dry Ratio of the Lung}

The wet/dry ratio of the control group was $5.5 \pm 0.4$. In the ischemic groups, the wet/dry ratio for the ischemia group was higher than that for the nitric oxide group $(7.36 \pm 1.35$ and $6.10 \pm 1.49$, respectively), but the difference was not significant. There was a significant difference between the control group and the ischemia group $(P<.05)$. This result suggests that addition of nitric oxide inhalation to ischemia mitigates lung edema that is otherwise easily generated by the conditions of BVAD support. 
TABLE 3. Oxygen consumption versus $\mathrm{Sv}_{2}$

\begin{tabular}{|c|c|c|c|c|c|c|c|c|}
\hline Group & Baseline & VAD set & $1 \mathrm{~h}$ & $2 \mathrm{~h}$ & $3 \mathrm{~h}$ & $4 \mathrm{~h}$ & $5 \mathrm{~h}$ & $6 \mathrm{~h}$ \\
\hline \multicolumn{9}{|l|}{ Control } \\
\hline $\mathrm{SvO}_{2}(\%)$ & $71.8 \pm 17.9$ & $76.3 \pm 10.2$ & $58.0 \pm 16.6$ & $55.5 \pm 14.4$ & $64.8 \pm 13.1$ & $62.3 \pm 7.3$ & $60.3 \pm 4.9$ & $60.3 \pm 4.9$ \\
\hline $\begin{array}{l}\text { Oxygen } \\
\text { extraction } \\
\text { ratio* }\end{array}$ & $0.26 \pm 0.18$ & $0.27 \pm 0.08$ & $0.29 \pm 0.09$ & $0.37 \pm 0.12$ & $0.37 \pm 0.09$ & $0.39 \pm 0.06$ & $0.39 \pm 0.04$ & $0.4 \pm 0.03$ \\
\hline \multicolumn{9}{|l|}{$\begin{array}{l}\text { Ischemia } \\
\text { only }\end{array}$} \\
\hline $\mathrm{SvO}_{2}(\%)$ & $80.0 \pm 4.8$ & $72.5 \pm 11.1$ & $65.5 \pm 12.0$ & $61.8 \pm 11.1$ & $66.1 \pm 9.5$ & $63.5 \pm 11.0$ & $61.8 \pm 11.9$ & $57.3 \pm 5.0$ \\
\hline $\begin{array}{l}\text { Oxygen } \\
\text { extraction } \\
\text { ratio* }\end{array}$ & $0.20 \pm 0.05$ & $0.27 \pm 0.06$ & $0.32 \pm 0.09$ & $0.30 \pm 0.07$ & $0.32 \pm 0.10$ & $0.37 \pm 0.09$ & $0.39 \pm 0.04$ & $0.42 \pm 0.14$ \\
\hline \multicolumn{9}{|l|}{ Nitric oxide } \\
\hline $\mathrm{SvO}_{2}(\%)$ & $85.2 \pm 3.4$ & $71.8 \pm 10.6$ & $72.6 \pm 3.8$ & $73.2 \pm 4.1$ & $72.6 \pm 6.7$ & $70.2 \pm 4.4$ & $65.6 \pm 5.0$ & $63.0 \pm 6.6$ \\
\hline $\begin{array}{l}\text { Oxygen } \\
\text { extraction } \\
\text { ratio* }\end{array}$ & $0.16 \pm 0.06$ & $0.24 \pm 0.03$ & $0.29 \pm 0.06$ & $0.27 \pm 0.07$ & $0.29 \pm 0.08$ & $0.30 \pm 0.07$ & $0.36 \pm 0.10$ & $0.36 \pm 0.07$ \\
\hline Phase & & Vf & Vf & $\begin{array}{l}\text { Weak } \\
\quad \text { contraction }\end{array}$ & $\begin{array}{l}\text { Weak } \\
\quad \text { contraction }\end{array}$ & $\begin{array}{l}\text { Weak } \\
\text { contraction }\end{array}$ & $\begin{array}{l}\text { RVAD } \\
\text { recover }\end{array}$ & No RVAD \\
\hline
\end{tabular}

*0xygen extraction ratio calculated as oxygen consumption (in milliliters per minute)/oxygen supply (in milliliters per minute).

\section{Discussion}

Postoperative right ventricular failure is observed in $20 \%$ to $30 \%$ of patients supported with an LVAD. Although the need for right ventricular assistance is temporary in many cases, some patients still require RVAD support because of inadequate response to vasodilators or other medicines. Therefore a management strategy for biventricular assistance that includes weaning the patient from RVAD support is an important issue. Furthermore, whereas common clinical VAD application involves a pulsatile pump, reports on management strategies for CFBVADs are rare. ${ }^{17-23}$

To maintain LVAD flow when cardiac function is critical, such as during Vf, it is generally understood that increasing preload supports LPF. This in turn means that an increased RPF is a necessary condition in a CFBVAD. Because increased RVAD flow may induce lung congestion, however, the avoidance of the latter becomes an important issue in the management of a CFBVAD. ${ }^{24}$

Meanwhile, nitric oxide inhalation therapy is often used in patients with pulmonary hypertension or acute respiratory distress syndrome 25,26 for its ability to dilate the pulmonary vascular bed. Because this process may reduce the operating frequency of RVAD support, investigators are inquiring with interest. In this series, inhalation of 40-ppm nitric oxide reduced PVR and PAP immediately, and it not only made easy an increase in RVAD flow but also maintained the circulations of the lungs and body without inducing lung edema, with the overall result of prompt weaning from RVAD support.

Nitric oxide inhalation therapy for patients with LVAD during right heart failure, where the VAD uses a pulsatile pump, is becoming a comparatively common procedure.
With the attention attracted to the clinical usefulness of the continuous-flow VAD in recent years, it is inevitable that combined use of the continuous-flow VAD and nitric will become known. Whereas nitric oxide inhalation has been recommended before implantation of an RVAD, nitric oxide inhalation in a case of BVAD implantation has seldom been examined. ${ }^{28}$

Some investigators have reported that the small amount of nitric oxide in coronary blood reduces the induction of nitric oxide synthase activity and its cytotoxicity in vitro. ${ }^{29-31}$ Our results showed that $\mathrm{LV} \mathrm{dp} / \mathrm{dt}$ and $\mathrm{RV} \mathrm{dp} / \mathrm{dt}$ decreased immediately after global ischemia, and the creatine phosphokinase MB isoenzyme level increased to similar degrees in both ischemic groups during the observation period, suggesting that a similar degree of ischemic myocardial injury was inflicted in both ischemic groups. Although this damage was occasionally fatal, significant recovery of cardiac function (to as much as $80 \%$ of control level) was observed in the nitric oxide group.

The fact that there was no time lag between the change of $\mathrm{LV} \mathrm{dp/dt} \mathrm{and} \mathrm{RV} \mathrm{dp/dt} \mathrm{is} \mathrm{encouraging.} \mathrm{Nevertheless,}$ nitric oxide inhalation did not directly affect the right ventricle but only reduced its afterload. On this issue, many studies have reported that right ventricular function is improved in response to nitric oxide inhalation mainly as a result of reduced pulmonary resistance, rather than any change in right ventricular contractility. ${ }^{32,33}$

RVEDP showed a similar increasing pattern in both ischemic groups, and it remained at almost same value as that in the control group except during the ischemic phase. Nevertheless, LV dp/dt, RV dp/dt, and LVEDP showed different patterns. This suggests that continuous injection of 
Ringer's lactate solution led to similar preloads in the groups and did not directly affect the function of the right side of the heart.

Because these differences were observed independent of the pump flows (which were maintained at similar levels in all groups), the possibility of a cardioprotective effect of NO inhalation therapy is suggested. However, because the degree of cardioprotective effect for cardiomyocytes could not be examined in our experiment by either a histologic or a tissue dynamic approach, we can only introduce the possibility and provide references. ${ }^{27,34-36}$

In this series, PAP increased after global ischemia. About this point, some facts should be observed. In the preceding experiment, despite increased LVAD speed, just before ventricular collapse occurred, it tended to be difficult to secure LVAD flow after global ischemia to maintain body circulation. To maintain LPF, security of the RPF was indispensable as preload of LVAD. However, despite increased RVAD speed just before atrial collapse occurred, it tended to be difficult to secure sufficient RPF after global ischemia. In this series, continuous injection of Ringer's lactate solution was performed to secure preload of the RVAD. For this reason, the RPF tend to increase a little after global ischemia.

On the other hand, although there was no significant difference between each group, $\mathrm{PaO}_{2}$ showed some decrease in all cases at equipping BVAD and when reddening of lungs was observed in the ischemia group after global ischemia. Because of these findings, a possibility that acute respiratory distress syndrome was induced at these period was also considered. However, in this series the clear parameter change proving existence of acute respiratory distress syndrome was not observed. Thus it is thought that PAP was increased after global ischemia according to these complex causes.

Moreover, the pattern of weaning from RVAD support by NO inhalation observed in this series may be transient, and this study does not exclude the possible existence of a rebound effect. To evaluate these concerns, this research is expected to be pursued in a longer term experiment.

Although various adverse effects of nitric oxide on patients and the environment have been reported, ${ }^{25-27}$ no adverse effects of nitric oxide inhalation, such as methemoglobinemia, were demonstrated in this series. Moreover, despite some reports that lung edema is induced by nitric oxide inhalation in healthy adults, there was no lung edema in the nitric oxide inhalation group in this experimental series. Rather, lung edema induced through CFBVAD support in the ischemic groups was improved by inhalation of nitric oxide. Thus we consider nitric oxide inhalation to reduce PVR, allowing a smoother lung circulation.

Incidentally, in this series spontaneous urination was observed in the nitric oxide group but not in the control group. Although assessment of this phenomenon is somewhat difficult, we believe that maintenance of the microcirculation of the urinary system leads to preservation of the voiding system.

From these results, the following hypothetical strategies are presumed to aid in the medical treatment of severe heart failure. In severe heart failure that requires strict mechanical support, to plan sufficient unloading of the whole myocardium, a patient should be equipped not only with LVAD but also with RVAD from an initial stage. However, in a postoperative early stage, in the state with the inadequate contractility of the heart, such as the Vf and weak contraction phases observed in this series, because RPF has to be increased for LPF reservation, nitric oxide inhalation from an early stage is used to reduce PVR. Sufficient unloading without lung edema by BVAD support becomes possible, and recovery of the cardiac function is promoted as a result. With recovery of the heartbeat, when sufficient output of right side circulation can be secured, weaning from the RVAD is started.

Although the RVAD was deployed through a thoracotomy in this study, taking into consideration the promotion of weaning from RVAD support expected through inhalation of nitric oxide, temporary right heart assistance may be achievable by a percutaneous approach. ${ }^{37-39}$ Although such equipment is devised to lessen operation stress by inserting cannula percutaneously into the right side circulation, the limitation of the flow depending on the diameter of cannula or the danger of the infection with long-term use are regarded as questionable.

Considering the possibility of the reduced RVAD flow and shortened supporting period by nitric oxide inhalation, it is thought that we can chose these mechanical support devices as one of the medical treatment strategies. An evaluation of this situation is expected in the course of future research.

\section{Conclusion}

In an acute, severe biventricular failure porcine model, nitric oxide inhalation under CFBVAD support significantly decreased PVR and reduced the afterload of the right ventricle. Consequently, the blood volume of the cardiopulmonary circulation under CFBVAD plus nitric oxide support increased without lung edema. Nitric oxide inhalation therapy has the potential to enable weaning from continuous flow RVAD support of animals with severe heart failure necessitating CFBVAD.

\section{References}

1. Kitamura M, Aomi S, Hachida M, Nishida H, Endo M, Koyanagi H. current strategy of temporary circulatory support for severe cardiac failure after operation. Ann Thorac Surg. 1999;68:662-5.

2. Lipsitz EC, Weinstein S, Stolar CJ. Endogenous nitric oxide and pulmonary vascular tone in the neonate. J Pediatr Surg. 1996;31:13740. 
3. Fierobe L, Brunet F, Dhainaut JF, Monchi M, Belghith M, Mira JP, et al. Effect of inhaled nitric oxide on right ventricular function in adult respiratory distress syndrome. Am J Respir Crit Care Med. 1995;151: 1414-9.

4. Journois D, Pouard P, Mauriat P. Inhaled nitric oxide as a therapy for pulmonary hypertension after operations for congenital heart defects. J Thorac Cardiovasc Surg. 1994;197:1129-35.

5. Rich GF, Murphy GD Jr, Roos CM. Inhaled nitric oxide: selective pulmonary vasodilation in cardiac surgical patients. Anesthesiology. 1993;78:1028-35.

6. Chang JC, Sawa Y, Matsuda H. Hemodynamic effect of inhaled nitric oxide in dilated cardiomyopathy patients on LVAD support. ASAIO J. 1997;43:M418-21.

7. Wagner F, Dandel M, Gunther G, Loebe M, Schulze-Neick I, Laucke $\mathrm{U}$, et al: Nitric oxide inhalation in the treatment of right ventricular dysfunction following left ventricular assist device implantation. Circulation. 1997;96(9 Suppl):II291-6.

8. Yahagi N, Kumon K, Takamoto S. Inhaled nitric oxide for the management of acute right ventricular failure in patients with a light ventricular assist system. Artif Organs. 1996;19:557-8.

9. Salamonsen RF, Kaye D, Esmore DS. Inhalation of nitric oxide provides selective pulmonary vasodilatation, aiding mechanical cardiac assist with Thoratec left ventricular assist device. Anaesth Intens Care. 1994;22:209-10.

10. Araki K, Anai H, Oshikawa M, Nakamura K, Onitsuka T. In vitro performance of a centrifugal, a mixed flow, and an axial flow blood pump. Artif Organs. 1998;22:366-70.

11. Oshikawa M, Araki K, Anai H, Nakamura K, Satoh M, Onitsuka T. In vivo evaluation of novel control method for a continuous-flow artificial heart. Jpn J Artif Organs. 1999;28:320-5.

12. Oshikawa M, Araki K, Nakamura K, Anai H, Onitsuka T. Detection of total assist and sucking points based on pulsatility of continuous-flow artificial heart: in vivo evaluation. ASAIO J. 1998;44:M704-7.

13. Araki K, Oshikawa M, Onitsuka T, Nakamura K, Anai H, Yoshihara $\mathrm{H}$. Detection of total assist and sucking points based on pulsatility of continuous-flow artificial heart: in vitro evaluation. ASAIO J. 1998; 44:M708-11.

14. Endo G, Araki K, Oshikawa M, Kojima K, Saitoh T, Nakamura K, et al. Control strategy for biventricular assistance with mixed-flow pump. Artif Organs. 2000;24:594-9.

15. Araki K, Oshikawa M, Anai H, Nakamura K, Sato M, Onitsuka T. detection and implication of the characteristic points for estimating the assisting condition in a continuous-flow artificial heart-in vitro study. Jpn J Artif Organs. 1999;28:383-93.

16. Edagawa M, Yoshida E, Matsuzaki Y, Shibuya K, Onitsuka T, Maruyama M. Reduction of post-ischemic lung reperfusion injury by fibrinolytic activity suppression. Transplantation. 1999;67:944-9.

17. Yuhki A, Hatoh E, Takatani S. Detection of suction and regurgitation of the implantable centrifugal pump based on the motor current waveform analysis and its application to optimization of pump flow. Artif Organs. 1999;23:532-7.

18. Savage EB, Clark RF, Magovern GJ. The AB-180 circulatory support system: summary of development and plans for phase I clinical trial. Ann Thorac Surg. 1999;68:768-74.

19. Noon GP, Lafuente JA, Irwin SI. Acute and temporary ventricular support with BioMedicus centrifugal pump. Ann Thorac Surg. 1999; 68:650-4.

20. Iijima T, Inamoto T, Nogawa M, Takatani S. Control of centrifugal blood pump based on the motor current. Artif Organs. 1997;21:655-60.

21. Amin DV, Antaki JF, Litwak P, Thomas D, Wu Z, Yu YC. Controller for an axial-flow blood pump. Biomed Instrum Technol. 1997;31: 483-7.

22. Nosé Y, Nakata K, Yoshikawa M, Letsou GV, Fujisawa A, Wolner E, et al. Development of a totally implantable biventricular bypass centrifugal blood pump system. Ann Thorac Surg. 1999;68:775-9.
23. Nakazawa T, Makinouchi K, Ohara Y, Ohtsubo S, Kawahito K, Tasai $\mathrm{K}$, et al. Development of a pivot bearing supported sealless centrifugal pump for ventricular assist. Artif Organs. 1996;20:485-90.

24. Nonaka $\mathrm{K}$, Linnewebwe J, Ishikawa S, Kawahito S, Motomura T, Ishitoya $\mathrm{H}$, et al. Assessing the calf pulmonary function during a long term BVAD study with a centrifugal blood pump. Aug 17-20, 2001; Seattle; 9th Congress of the International Society for Rotary Blood Pumps.

25. Muller W, Kachel W, Lasch P, Varnholt V, Konig S. Inhaled nitric oxide during extracorporeal membrane oxygenation for the treatment of severe persistent pulmonary hypertension of the newborn. Artif Organs. 1996;20:60-3.

26. Young JD, Sear JW, Valvini EM. Kinetics of methaemoglobin and serum nitrogen oxide production during inhalation of nitric oxide in volunteers. Br J Anaesth. 1996;76:652-6.

27. Hayward CS, Kelly RP, Macdonald PS. Inhaled nitric oxide in cardiology practice. Cardiovasc Res. 1999;43:628-38.

28. Chang JC, Sawa Y, Ohtake S, Fukushima N, Nishimura M, Kagizaki $\mathrm{K}$, et al. Hemodynamic effect of inhaled nitric oxide in dilated cardiomyopathy patients on LVAD support. ASAIO J. 1997;43:M418-21.

29. Yang XP, Liu YH, Shesely EG, Bulagannawar M, Liu F, Carretero OA. Endothelial nitric oxide gene knockout mice: cardiac phenotypes and the effect of angiotensin-converting enzyme inhibitor on myocardial ischemia/reperfusion injury. Hypertension. 1999;34:24-30.

30. Sato H, Zhao ZQ, Jordan JE. Basal nitric oxide expresses endogenous cardioprotection during reperfusion by inhibition of neutrophil-mediated damage after surgical revascularization. J Thorac Cardiovasc Surg. 1997;113:399-409.

31. Stamler JS, Loh E, Roddy MA. Nitric oxide regulates basal systemic and pulmonary vascular resistance in healthy humans. Circulation. 1994;89:2035-40.

32. Cheifetz IM, Craig DM, Kern FH, Black DR, Hillman ND, Greeley WJ, et al. Nitric oxide improves transpulmonary vascular mechanics but does not change intrinsic right ventricular contractility in an acute respiratory distress syndrome model with permissive hypercapnia. Crit Care Med. 1996;24:1554-61.

33. Hillman ND, Cheifetz IM, Craig DM, Smith PK, Ungerleider RM, Meliones JN. Inhaled nitric oxide, right ventricular efficiency, and pulmonary vascular mechanics: selective vasodilation of small pulmonary vessels during hypoxic pulmonary vasoconstriction. J Thorac Cardiovasc Surg. 1997;113:1006-13.

34. Pabla R, Curtis MJ. Endogenous protection against reperfusion-induced ventricular fibrillation: role of neuronal versus non-neuronal sources of nitric oxide and species dependence in the rat versus rabbit isolated heart. J Mol Cell Cardiol. 1996;28:2097-110.

35. Sato H, Zhao ZQ, Jordan JE, Todd JC, Riley RD, Taft CS, et al. Basal nitric oxide expresses endogenous cardioprotection during reperfusion by inhibition of neutrophil-mediated damage after surgical revascularization. J Thorac Cardiovasc Surg. 1997;113:399-409.

36. Vinten-Johansen J, Zhao ZQ, Nakamura M, Jordan JE, Ronson RS, Thourani VH, et al. Nitric oxide and the vascular endothelium in myocardial ischemia-reperfusion injury. Ann N Y Acad Sci. 1999;874: 354-70.

37. Yano M, Matsuo K, Hatane T, Araki K, Onitsuka T, Shibata K, et al. The feasibility and efficacy of right ventricular assistance without thoracotomy. ASAIO J. 1993;29:120-5.

38. Yano M, Onitsuka T, Shibata K, Koga Y. efficacy and safety of a percutaneous right ventricular assist system. Ann Thorac Surg. 1996; 61:1231-5.

39. Matsuo K, Yano M, Nakamura K, Onitsuka T. Potentialities and problems of a novel bilateral ventricular assist system without thoracotomy. Artif Organs. 2000;24:148-55. 